\title{
The Effect of Atopy on the Incidence of Osteitis in Patients with Chronic Rhinosinusitis
}

\author{
Nur Saadah Mohamad (D)', Sakinah Mohamad (D)', Mohd Ezane Aziz², Baharudin Abdullah' \\ 'Department of Otorhinolaryngology - Head \& Neck Surgery, School of Medical Sciences, Universiti Sains Malaysia, Kubang Kerian, Kelantan, I6I50, \\ Malaysia; ' 2 Department of Radiology, School of Medical Sciences, Universiti Sains Malaysia, Kubang Kerian, I6I50, Kelantan, Malaysia \\ Correspondence: Baharudin Abdullah, Department of Otorhinolaryngology - Head and Neck Surgery, School of Medical Sciences, Universiti Sains \\ Malaysia, Kubang Kerian, 16150, Kelantan, Malaysia, Tel +60976764I6, Email baharudin@usm.my
}

Background: Atopy may not contribute directly to the pathogenesis of chronic rhinosinusitis (CRS) and could be a coexisting disease, but it may play a disease-modifying role in CRS. The aim of this study was to determine the effect of atopy on the incidence of osteitis in patients with CRS.

Methods: A cross-sectional study at a tertiary center was conducted. Computed tomography of paranasal sinuses (CTPNS) of 75 CRS patients was analyzed. Skin prick test was used to determine the atopy among the CRS patients. The evaluation consisted of symptom score, Lund-Kennedy endoscopic score and CTPNS assessment by Lund Mackay (LM) staging system and Global Osteitis Scoring Scale (GOSS).

Results: About $54.7 \%$ of CRS patients had atopy $(n=41)$, and $64 \%(n=48)$ had osteitis changes. No significant difference $(p>0.05)$ was found for symptom and endoscopic scores in atopic and non-atopic CRS. Atopic and non-atopic CRS patients demonstrated comparable LM and GOSS scores (both $p>0.05$ ). Logistic regression analysis revealed that gender, nasal polyps and bronchial asthma were significantly associated with the incidence of osteitis. Significant correlation was found between LM and GOSS scores in atopic CRS ( $\mathrm{r}=0.81, p<0.05)$. Correspondingly, both scores were found to be significantly correlated in non-atopic $\mathrm{CRS}(\mathrm{r}=0.74, p<0.05)$.

Conclusion: Atopic sensitization has no effect on the incidence and severity of osteitis in patients with CRS. The present study suggests that osteitis occurs independently from the atopic pathway.

Keywords: chronic rhinosinusitis, osteitis, atopy, computed tomography, Lund Mackay, Global Osteitis Scoring Scale

\section{Introduction}

Inflammation not only involves mucosa in chronic rhinosinusitis (CRS) but also extends to the underlying paranasal sinus bone where it causes osteitis. ${ }^{1}$ Osteitis is an inflammation affecting bone without marrow space due to the deposition of the inflammatory cytokines inside the underlying bone. ${ }^{2}$ On computed tomography assessment, mucosal thickening and polyps appeared as homogeneous opacification in the paranasal sinuses while the osteitis changes appeared as heterogeneous, irregular bony thickening together with an increase of the bone density. ${ }^{1,3}$ Osteitis has been shown to correspond to substantial endoscopic severity and poorer computed tomography grading. ${ }^{2,4}$ It has been recognized as a poor prognostic factor in the surgical management of CRS. ${ }^{2}$ Following endoscopic sinus surgery, retained osteitic bone may act as a focal point to induce inflammation on the surrounding mucosa which may lead to recurrence. ${ }^{5}$ Prolonged antibiotic use to treat the bony inflammation similar to treatment for osteomyelitis together with radical and extensive surgery requiring drilling and removal of the osteitis bone are among the advocated treatment to improve the outcome. ${ }^{6}$

Even though the role of atopy in the pathogenesis of chronic rhinosinusitis had been investigated, its role in the outcome of management is inconclusive. Some studies suggested atopy causes more severe symptoms, a higher computed tomography score and poorer surgical outcomes but some other studies failed to establish a clear relationship between the severity of CRS with the atopic status of the patient. ${ }^{7-9}$ Whilst the effect of atopy on osteitis changes in patients with CRS is not well understood, a prior study showed significant bone remodeling occurs in CRS patients with concomitant allergy implying that atopy may play a role in osteitis. ${ }^{1}$ The aforementioned study performed a histopathological evaluation 
of the presence of osteitis in ethmoidal bone for both atopic and non-atopic patients with CRS. The study found the incidence of osteitis was higher in the atopic compared to non-atopic CRS patients. With this finding, it is plausible to hypothesize that atopy is a significant risk factor in the incidence of osteitis in CRS patients. Thus, the aim of this study was to determine the effect of atopy in the incidence of osteitis amongst CRS patients.

\section{Methods}

A cross-sectional study was conducted in a tertiary hospital from April 2020 until April 2021. Ethical clearance was obtained from the Human Research Ethics Review Board, Universiti Sains Malaysia (No: USM/JEPeM/19120843) and was performed in adherence with the Declaration of Helsinki. All patients provided informed consent before enrolment.

\section{Study Population}

Consecutive adult patients above age 18 years with the diagnosis of CRS with or without polyps according to the European position paper on rhinosinusitis and nasal polyp ${ }^{10}$ who had computed tomography of paranasal sinuses (CTPNS) examination were included. The exclusion criteria were patients with prior sinus surgery, trauma to the skull base or facial bone, sinonasal malignancy, craniofacial abnormality and pregnancy. The CTPNS was retrieved from the radiology information system and picture archive communication (PAC) system. The CTPNS images were acquired from SOMATOM $^{\circledR}$ Definition AS+ (Siemens Healthcare GmbH, Germany) which can produce 128 slices of images per rotation. The paranasal sinuses were assessed from serial images (1 mm slices) on coronal, axial and sagittal views on both right and left sides. Evaluation by CTPNS within 3 months prior or after allergy assessment was included. The radiological assessments were done by using Lund Mackay (LM) system and Global Osteitis Scoring Scale (GOSS). ${ }^{5,11}$ The radiological evaluation was performed by the first author (N.S.M.), a radiologist (M.E.A.) and a rhinologist (B.A.).

\section{Atopy Status}

Clinical history for allergy and atopy (allergic rhinitis, eczema and asthma) was obtained from all patients prior to skin prick test (SPT). Skin prick test was done on each patient before treatment or 2 weeks after refraining from oral or intranasal corticosteroid and oral antihistamines. Skin prick test was performed on the patient's volar forearm using the common inhalant and food allergens in our patients. Histamine was used for positive control and saline for negative control. A positive skin test result was defined as a wheal of more than $3 \mathrm{~mm}$ to the allergens with a non-reactive negative control after 20 minutes. ${ }^{12}$ Patients with wheal size of less than $3 \mathrm{~mm}$ with positive allergy and atopy history underwent venous blood taking for serum specific IgE level to Dermatophagoides and Blomia tropicalis (analyzed via ImmunoCAP, Phadia AB, Uppsala, Sweden). A value of $0.35 \mathrm{KU} / \mathrm{L}$ or more of the serum specific IgE was considered positive for atopy. Patients with either a positive SPT or serum specific IgE were considered as having atopy. Asthma status was defined as a prior diagnosis of airway hyper-responsiveness or requiring regular beta-agonist or corticosteroid inhalers. Smokers were defined as any patient currently smoking or who had ceased within the last 12 months. Aspirin sensitivity was defined as a history of skin reaction, rhinitis or asthma induced condition due to consumption of aspirin or non-steroidal anti-inflammatory drugs (NSAIDs).

\section{Symptom and Endoscopic Assessments}

A five-item symptom score was used for patients to rate the severity of their symptoms. ${ }^{4}$ Symptoms of nasal blockage, headache/facial pain, thick nasal discharge, postnasal drip and smell or taste loss were rated using Likert scale as follows: 0 (no symptom), 1 (very mild symptom), 2 (mild), 3 (moderate), 4 (severe) and 5 (very severe). The Lund-Kennedy endoscopic scoring system ${ }^{13}$ was used to grade the severity of the nasal polyp. The scoring comprised size of polyp $(0=$ no polyp, $1=$ polyp till middle meatus, $2=$ polyp extend beyond middle meatus $)$, edema of turbinates $(0=$ absent, $1=$ mild to moderate edema, $2=$ polypoidal turbinate $)$ and presence of discharge $(0=$ none, $1=$ clear and thin, $2=$ thick and purulent). The total maximum score was 12 . 


\section{Radiological Measurements}

The LM score system was used to evaluate the mucosa thickening of the sinuses in axial and coronal views. The right and left sinuses were respectively divided into six portions, including maxillary sinus, anterior ethmoid sinus, posterior ethmoid sinus, sphenoid sinus, frontal sinus and ostiomeatal complex. The severity of sinus mucosal inflammation or fluid accumulation in paranasal sinus was scored as 0 (complete lucency), 1 (partial lucency) and 2 (complete opacity) (Figure 1). Score for the ostiomeatal complex was graded as 0 for no obstruction or 2 for an obstruction. The total maximum score was 24 .

Osteitis was defined as loss of bone definition, hyperostosis, new bone formation or signal heterogeneity overlying each sinus wall. ${ }^{5}$ The grading per sinus was scored according to GOSS as grade 1 (less than $50 \%$ of the sinus wall involved with $<3 \mathrm{~mm}$ wide osteitis), grade 2 (less than $50 \%$ of the sinus wall involved with 3-5 $\mathrm{mm}$ wide osteitis), grade 3 (less than $50 \%$ of the sinus involved with more than $5 \mathrm{~mm}$ wide osteitis or greater than $50 \%$ of the sinus wall involved with $<3 \mathrm{~mm}$ wide osteitis), grade 4 (greater than $50 \%$ of the sinus wall involved with $3-5 \mathrm{~mm}$ wide osteitis) and grade 5 (greater than $50 \%$ of the sinus wall involved with osteitis thicker than $5 \mathrm{~mm}$ wide). The hyperostotic focus was identified and measured using a digital measurement tool in the PAC system. Evaluation was performed for frontal, anterior ethmoid, posterior ethmoid, sphenoid and maxillary sinuses on both sides (Figure 2). Measurement for the anterior and posterior ethmoidal air cells was taken on an axial view following the identification of the basal lamella. Measurement for the sphenoid sinus was done at mid-sphenoid in an axial view. Measurement for the maxillary sinus was done at the area posterior to crista galli in coronal view. Measurement for the frontal sinus was done at mid-frontal area at the level of nasofrontal beak in sagittal view. The total 10 sinuses score were added with a maximum score of 50 . A total score of 5 was not significant, 5-20 was mild, 20-35 was moderate and more than 35 was severe osteitis.

\section{Statistical Analysis}

Data were entered and analyzed using IBM SPSS Statistics for Windows, Version 22.0 (IBM Corp, Armonk, NY, USA). Descriptive analysis was used for patients' demographic data and they were presented as mean, standard deviation (SD) and frequency. The distribution of CRS patients with osteitis changes, distribution of osteitis severity and distribution of skin prick test results were presented as a frequency. Numerical data for the symptom score, endoscopic score and CT score (both LM and GOSS scores) were presented as mean with standard deviation (SD) and calculated by Mann-
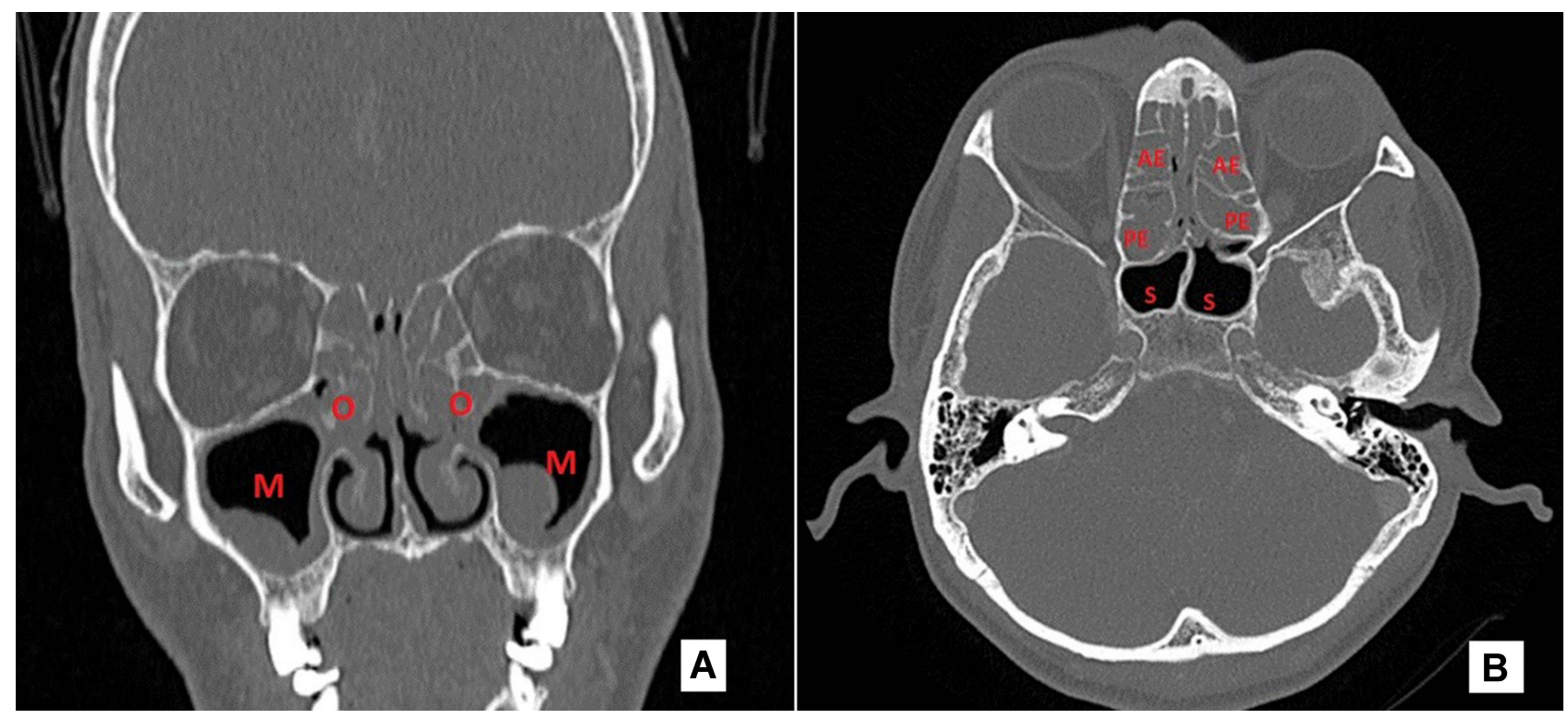

Figure I Radiological measurement using Lund Mackay score system for CTPNS axial (A) and coronal (B) sections, for example (A) the right maxillary sinus was scored as I, left maxillary sinus as I and both ostiomeatal complexes as 2 each and (B) the anterior and posterior ethmoidal air cells on both sides were scored as 2 each and both sphenoid air cells as 0 each.

Abbreviations: M, maxillary sinus; O, ostiomeatal complex; $\mathrm{AE}$, anterior ethmoid sinus; $\mathrm{PE}$, posterior ethmoid sinus; $\mathrm{S}$, sphenoid sinus. 


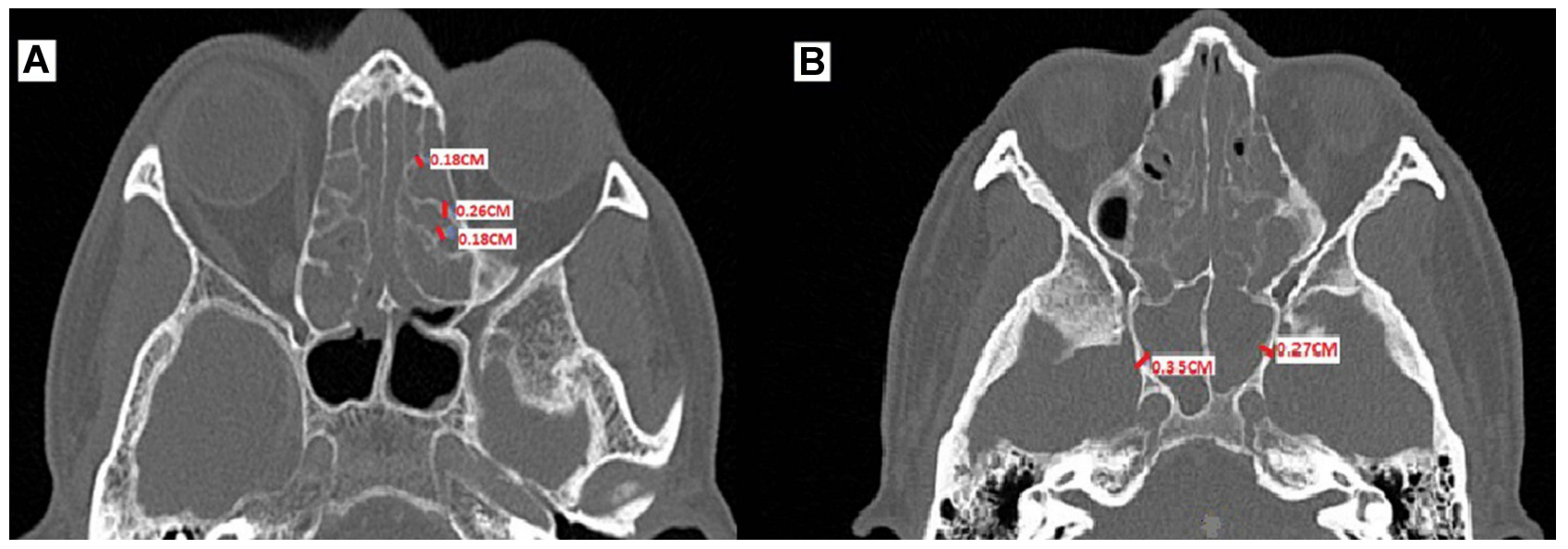

Figure 2 Radiological measurement using Global Osteitis score scale system for computed tomography of the paranasal sinuses. Measurement was made for hyperostosis area with maximal thickness of the osteitic focus in all the sinuses, for example (A) ethmoidal sinus and (B) sphenoid sinus.

Whitney test to compare the differences. The association between atopy status and CT scores (in both LM and GOSS scores) was determined by Mann-Whitney test. Pearson correlation coefficient was used to determine the association of disease severity according to LM score and GOSS score in atopic and non-atopic CRS. The association of individual factors such as age, atopy, polyps, gender, smoking, bronchial asthma and NSAIDs sensitivity with osteitis was evaluated by logistic regression analysis. The statistical significance was set at $p<0.05$.

\section{Results}

\section{Patient Population}

Seventy-five patients with CRS were enrolled. The demographic characteristics of the patients are shown in Table 1. The mean age for atopic CRS patients was $41.95 \pm 12.05$ and non-atopic CRS was $49.76 \pm 13.71$. Male was the predominant gender in each group. There was a higher number of concomitant bronchial asthma in the atopic group (79.41\%) but an equal number of NSAIDs sensitivity in both atopic and non-atopic CRS. The results of the SPT are shown in Figure 3.

Table I Patients' Demographic Data

\begin{tabular}{|c|c|c|c|}
\hline \multirow[t]{2}{*}{ Variables } & \multicolumn{2}{|c|}{ Chronic Rhinosinusitis, n (\%) } & \multirow[t]{2}{*}{$p$ value } \\
\hline & Non-Atopic $(n=34)$ & Atopic $(n=4 I)$ & \\
\hline Age (years, mean $\pm S D$ ) & $49.76 \pm|3.7|$ & $41.95 \pm 12.05$ & $0.008^{\mathrm{a}}$ \\
\hline \multicolumn{4}{|l|}{ Gender } \\
\hline Female & $16(47.06 \%)$ & $14(34.15 \%)$ & $0.256^{\mathrm{b}}$ \\
\hline Male & I8 (52.94\%) & 27 (65.85\%) & \\
\hline \multicolumn{4}{|l|}{ Smoking } \\
\hline No & $24(70.59 \%)$ & $28(68.29 \%)$ & $0.830^{\mathrm{b}}$ \\
\hline Yes & $10(29.41 \%)$ & $13(3 \mid .71 \%)$ & \\
\hline \multicolumn{4}{|l|}{ Bronchial asthma } \\
\hline No & $27(79.41 \%)$ & $26(63.41 \%)$ & $0.130^{\mathrm{b}}$ \\
\hline Yes & 7 (20.59\%) & $15(36.59 \%)$ & \\
\hline \multicolumn{4}{|l|}{ Sensitivity to NSAIDs } \\
\hline No & $33(97.06 \%)$ & 40 (97.56\%) & $0.705^{c}$ \\
\hline Yes & I (2.94\%) & I (2.44\%) & \\
\hline
\end{tabular}

Notes: ${ }^{\mathrm{a}}$ Mann-Whitney test, ${ }^{\mathrm{b}}$ Pearson chi-square test, ${ }^{\mathrm{c}}$ Fisher's exact test. 


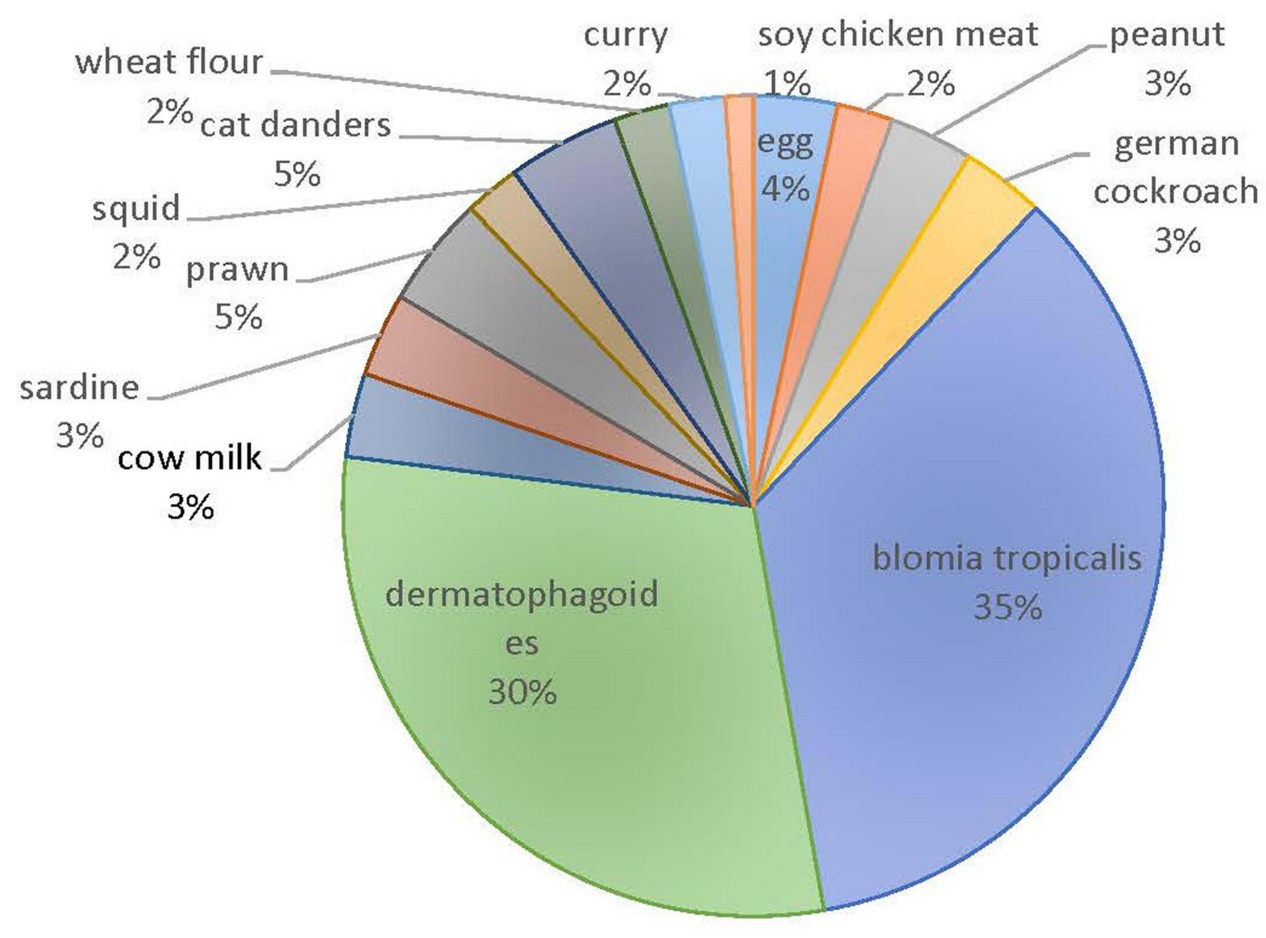

Figure 3 Distribution of skin prick test result.

\section{Symptom and Endoscopy Scores}

The mean symptom score was higher in non-atopic compared to atopic CRS patients but the difference was not significant (Table 2). The symptoms of nasal blockage, headache or facial pain, smell or taste loss, postnasal drip and thick nasal discharge were not much difference in both groups, but postnasal drip was slightly higher in the atopic group. Likewise, the mean endoscopic score in both groups was not significantly different (Table 2).

\section{Radiological Evaluation}

Radiographic evidence of disease severity as classified by LM score correlates with an elevated GOSS in both atopic and non-atopic CRS (Table 2). Atopic status did not impact radiographic disease severity or osteitis severity, as atopic and non-atopic CRS patients demonstrated comparable LM score and GOSS score (both $p>0.05$ ). The highest LM score was

Table 2 The Symptoms, Endoscopic and Computed Tomographic Scores in Atopic and Non-Atopic Chronic Rhinosinusitis

\begin{tabular}{|c|c|c|c|}
\hline & \multicolumn{2}{|c|}{ Disease Severity (Mean \pm SD) } & \multirow[t]{2}{*}{$p$ value* } \\
\hline & Atopic Chronic Rhinosinusitis & Non-Atopic Chronic Rhinosinusitis & \\
\hline Total symptom score & $8.68 \pm 3.46$ & $9.62 \pm 4.61$ & 0.61 \\
\hline - Nasal block & $3.29 \pm 1.42$ & $3.76 \pm 1.74$ & 0.05 \\
\hline - Headache/facial pain & $0.88 \pm 1.27$ & $1.09 \pm 1.56$ & 0.85 \\
\hline - Smell/taste loss & $1.22 \pm 1.59$ & $I .4 I \pm I .76$ & 0.62 \\
\hline - Postnasal drip & $0.80 \pm 1.35$ & $0.62 \pm 1.16$ & 0.49 \\
\hline - Thick nasal discharge & $2.49 \pm 1.52$ & $2.74 \pm 1.52$ & 0.44 \\
\hline Lund-Kennedy endoscopic score & $5.34 \pm 1.62$ & $5.29 \pm 2.18$ & 0.95 \\
\hline Lund Mackay score & $8.88 \pm 5.22$ & $9.06 \pm 5.01$ & 0.68 \\
\hline Global Osteitis Scoring Scale & $10.90 \pm 10.50$ & $9.03 \pm 7.83$ & 0.71 \\
\hline
\end{tabular}

Note: *Mann-Whitney test. 
at the anterior ethmoid sinus $(2.12 \pm 0.95)$ in non-atopic CRS while the lowest was at the sphenoid sinus $(0.88 \pm 1.09)$ in atopic CRS (Table 3). The greatest osteitis changes was at the maxillary sinus (2.95 \pm 2.70$)$ in atopic CRS while the least osteitis was at the posterior ethmoid sinus (1.15 \pm 1.58$)$ in non-atopic CRS (Table 3). Significant correlation was found between LM and GOSS scores in atopic CRS $(\mathrm{r}=0.81)$. Similarly, both scores were found to be significantly correlated in non-atopic CRS ( $\mathrm{r}=0.74)$. Mild and moderate osteitis were found in both atopic and non-atopic CRS but severe osteitis was found only in atopic CRS (Table 4).

\section{Relation of Atopy Status with Nasal Polyp}

The subgroup analysis of CRS patients with and without nasal polyps showed there was no difference in LM score between both groups (Table 5). Similarly, both groups demonstrated no significant difference in GOSS score.

\section{Factors Associated with Osteitis}

Logistic regression analysis revealed gender, nasal polyps and bronchial asthma were the individual factors significantly associated with the presence of osteitis (Table 6). Male patients, the presence of polyps and concomitant bronchial asthma have a significantly increased odds of developing osteitis.

Table 3 Lund Mackay Score and Global Osteitis Score Between Atopic and Non-Atopic Chronic Rhinosinusitis

\begin{tabular}{|c|c|c|c|}
\hline Sinus & $\begin{array}{c}\text { Non-Atopic Chronic Rhinosinusitis } \\
(\text { Mean } \pm \text { SD) }\end{array}$ & $\begin{array}{l}\text { Atopic Chronic Rhinosinusitis } \\
\text { (Mean士SD) }\end{array}$ & $p$ value* \\
\hline \multicolumn{4}{|c|}{ Lund Mackay score } \\
\hline Maxillary & $1.91 \pm 0.75$ & $1.80 \pm 0.75$ & 0.30 \\
\hline Anterior ethmoid & $2.12 \pm 0.95$ & $1.95 \pm 0.74$ & 0.33 \\
\hline Posterior ethmoid & $1.50 \pm 0.90$ & $1.66 \pm 0.91$ & 0.54 \\
\hline Frontal & $1.50 \pm 1.38$ & $1.07 \pm 1.27$ & 0.16 \\
\hline Sphenoid & $1.03 \pm 1.03$ & $0.88 \pm 1.09$ & 0.41 \\
\hline Ostiomeatal complex & $1.00 \pm 1.50$ & $0.98 \pm 1.56$ & 0.84 \\
\hline Total score & $9.06 \pm 5.01$ & $8.88 \pm 5.22$ & 0.68 \\
\hline \multicolumn{4}{|c|}{ Global Osteitis score } \\
\hline Maxillary & $2.79 \pm 2.10$ & $2.95 \pm 2.70$ & 0.85 \\
\hline Anterior ethmoid & $2.03 \pm 2.04$ & $2.88 \pm 2.51$ & 0.19 \\
\hline Posterior ethmoid & $1.15 \pm 1.58$ & $1.78 \pm 2.34$ & 0.44 \\
\hline Frontal & $\mid .74 \pm 2.5 \mathrm{I}$ & $1.66 \pm 2.71$ & 0.53 \\
\hline Sphenoid & $1.38 \pm 2.34$ & $1.63 \pm 2.63$ & 0.77 \\
\hline Total score & $9.03 \pm 7.83$ & $10.90 \pm 10.50$ & 0.71 \\
\hline
\end{tabular}

Note: *Mann-Whitney test.

Table 4 Distribution of Severity of Osteitis in Atopic and Non-Atopic Chronic Rhinosinusitis

\begin{tabular}{|l|c|c|}
\hline \multirow{2}{*}{ Global Osteitis Score } & \multicolumn{2}{|c|}{ Chronic Rhinosinusitis, n (\%) } \\
\cline { 2 - 3 } & Non-Atopic & Atopic \\
\hline Not significant (0-5) & $11(14.67 \%)$ & $16(21.33 \%)$ \\
Mild (5-20) & $20(26.67 \%)$ & $19(25.33 \%)$ \\
Moderate (20-35) & $3(4.00 \%)$ & $4(5.33 \%)$ \\
Severe (35-50) & $0(0.00 \%)$ & $2(2.67 \%)$ \\
\hline
\end{tabular}


Table 5 Comparison of Lund Mackay Score and Global Osteitis Score in Chronic Rhinosinusitis with and without Nasal Polyps

\begin{tabular}{|c|c|c|c|}
\hline & Non-Atopic (Mean \pm SD) & Atopic (Mean \pm SD) & $p$ value* \\
\hline \multicolumn{4}{|c|}{ Chronic rhinosinusitis with nasal polyps } \\
\hline Patients (n) & 23 & 19 & \\
\hline Lund Mackay & $10.83 \pm 4.93$ & $11.26 \pm 4.04$ & 0.79 \\
\hline Global Osteitis & $10.96 \pm 8.37$ & $16.16 \pm 10.59$ & 0.09 \\
\hline \multicolumn{4}{|c|}{ Chronic rhinosinusitis without nasal polyps } \\
\hline Patients (n) & 11 & 22 & \\
\hline Lund Mackay & $5.36 \pm 2.69$ & $6.82 \pm 5.32$ & 0.75 \\
\hline Global Osteitis & $5.00 \pm 4.67$ & $6.36 \pm 8.22$ & 0.98 \\
\hline
\end{tabular}

Note: *Mann-Whitney test.

\section{Discussion}

Osteitis was reported as one of the prognostic factors in the management of CRS patients and assessment of the CTPNS helps in the decision-making process of the need for surgical intervention and to identify the related anatomical structures that must be addressed. ${ }^{10}$ Measurement of the bony thickness of the sinus walls is widely used although there is no consensus on the exact degree of thickening for osteitis. Bone density assessment of the sinus walls' thickness can be made by Hounsfield unit or bone 99m-technetium-methylene diphosphonate single photon emission method, the latter being more expensive and time consuming. ${ }^{3,14}$

Lee et al assessed osteitis based on the thickness of bony partitions in maxillary, ethmoid and sphenoid sinuses which was classified as not significant $(<3 \mathrm{~mm})$, mild $(3 \mathrm{~mm})$, moderate $(4-5 \mathrm{~mm})$ and severe $(>5 \mathrm{~mm}) .{ }^{15}$ They found a higher score of LM in patients with osteitis changes, but the assessment did not include the frontal sinus. In contrast, another study measured osteitis change at 3 points (the posterolateral wall maxillary sinus, mean thickness of 3 randomly selected ethmoid septa and the midpoint of middle turbinate) and demonstrated CRS patients with allergy had significant osteitis on CTPNS. ${ }^{16}$ However, their measurements did not include the frontal and sphenoid sinuses. By incorporating the severity of osteitis in each of the sinuses and the number of sinuses involved, GOSS is able to reflect the severity and extent of osteitis and provides a more accurate assessment. ${ }^{5}$

Atopy may not contribute directly to the pathogenesis of CRS and could be a coexisting disease but it may play a disease-modifying role in CRS. Concomitant atopic conditions such as allergic rhinitis and bronchial asthma in patients having CRS are assumed to have poorer outcome due to their association with greater mucosal inflammation. ${ }^{8,17}$ However, the evidences for this link have been conflicting. A strong relationship was established in a study by Pearlman et al between severity of CRS with asthma and atopy. ${ }^{18}$ On the other hand, a study by Robinson et al showed no significant difference in the severity between atopic and non-atopic CRS patients on CT evaluation. ${ }^{7}$

In the present study, we found no significant difference in the CT score by LM and GOSS grading systems between atopic and non-atopic CRS. Additionally, logistic regression analysis confirmed atopy status did not have any impact on the presence of osteitis. Interestingly, we found male gender, the presence of polyps and concomitant bronchial asthma have increased odds of having osteitis. It is worth noting that all patients with NSAIDs sensitivity have osteitis. The connection between these factors with osteitis is not clear and further studies are required to determine their specific roles.

The correlation between LM and GOSS scores deserves further mention. The strong positive correlation between LM and GOSS scores indicates the link between severity of CRS with osteitis changes. The association of severe CRS with osteitic changes is related to higher prevalence of bony remodeling found in patients with higher LM score. Thus, the finding of this strong correlation in our study reaffirms the significant association of higher osteitic scores with greater severity of disease found in earlier studies. ${ }^{4,5,15,19}$

Osteitis changes led to persistence of disease and is a predictor of recalcitrant CRS whereas the presence of atopy brought about worse clinical and radiological burden of disease. ${ }^{6,8}$ As both conditions are associated with poor outcome, 
an appropriate and suitable treatment plan should be applied in managing such patients to prevent recurrence and improved quality of life. In a landmark histopathological study by Mutijima et al the occurrence of osteitis was connected with atopy. ${ }^{1}$ Osteitis changes in the ethmoidal bone of CRS patients subjected to surgery was compared between two CRS groups with or without atopy. They observed that all CRS patients having atopy had osteitis changes compared to those without atopy. This remarkable finding attests to the association between atopy and occurrence of osteitis. A comparative study was conducted by $\mathrm{Zhu}$ et al in patients requiring endoscopic sinus surgery to determine the characteristics between eosinophilic and non-eosinophilic CRS phenotypes. ${ }^{20}$ Even though they found the occurrence of nasal polyps, bronchial asthma and atopy was significantly higher in eosinophilic CRS, the osteitis score was significantly worse in non-eosinophilic CRS. This finding implies that atopy does not influence the incidence and severity of osteitis in patients with CRS and other factors could be involved. The development of osteitis might be due to multiple factors and this would explain the conflicting findings.

\section{Limitations}

The cross-sectional design of this study represents an evaluation at one point of time of the CT scan but the CT features may depend on the season, disease state and medications, and future studies are recommended to look at these changes over a period of time.

\section{Conclusions}

Atopic sensitization has no effect on the incidence and severity of osteitis in patients with CRS. The present study suggests that osteitis occurs independently from the atopic pathway.

\section{Ethics Approval and Informed Consent}

All procedures performed and data collected in this study adhered to the guidelines of the Declaration of Helsinki and the ethical standards of institutional ethics review committees. The Human Research Ethics Review Board of Universiti Sains Malaysia (JEPeM Code: No: USM/JEPeM/19120843) granted approval to conduct this research. Written consent was obtained from each patient.

\section{Author Contributions}

All authors made substantial contributions to conception and design, acquisition of data, or analysis and interpretation of data; took part in drafting the article or revising it critically for important intellectual content; agreed to submit to the current journal; gave final approval for the version to be published; and agreed to be accountable for all aspects of the work.

\section{Disclosure}

The authors declare no conflicts of interest in this work.

\section{References}

1. Mutijima E, Delvenne P, El-Shazly AE. Osteitis in chronic rhinosinusitis: a histopathological study of human ethmoid bone remodeling in allergic versus non-allergic chronic rhinosinusitis. Adv Cell Mol Otolaryngol. 2014;2(1):23504. doi:10.3402/acmo.v2.23504

2. Bhandarkar ND, Mace JC, Smith TL. The impact of osteitis on disease severity measures and quality of life outcomes in chronic rhinosinusitis. Int Forum Allergy Rhinol. 2011;1(5):372-378. doi:10.1002/alr.20068

3. Emre IE, Celebi I, Ercan I. The radiologic evaluation of osteitis type and formation in chronic rhinosinusitis with and without nasal polyposis. Am $J$ Rhinol Allergy. 2015;29(6):e201-204. doi:10.2500/ajra.2015.29.4240

4. Snidvongs K, McLachlan R, Sacks R, et al. Correlation of the Kennedy Osteitis Score to clinico-histologic features of chronic rhinosinusitis. Int Forum Allergy Rhinol. 2013;3(5):369-375. doi:10.1002/alr.21113

5. Georgalas C, Videler W, Freling N, et al. Global Osteitis Scoring Scale and chronic rhinosinusitis: a marker of revision surgery. Clin Otolaryngol. 2010;35(6):455-461. doi:10.1111/j.1749-4486.2010.02218.x

6. Bhandarkar ND, Sautter NB, Kennedy DW, et al. Osteitis in chronic rhinosinusitis: a review of the literature. Int Forum Allergy Rhinol. 2013;3 (5):355-363. doi:10.1002/alr.21118

7. Robinson S, Douglas R, Wormald PJ. The relationship between atopy and chronic rhinosinusitis. Am J Rhinol. 2006;20(6):625-628. doi:10.2500/ ajr.2006.20.2907 
8. Abdullah B, Vengathajalam S, Md Daud MK, et al. The clinical and radiological characterizations of the allergic phenotype of chronic rhinosinusitis with nasal polyps. $J$ Asthma Allergy. 2020;13:523-531. doi:10.2147/JAA.S275536

9. Hamizan AW, Loftus PA, Alvarado R, et al. Allergic phenotype of chronic rhinosinusitis based on radiologic pattern of disease. Laryngoscope. 2018;128(9):2015-2021. doi:10.1002/lary.27180

10. Fokkens WJ, Lund VJ, Hopkins C, et al. European position paper on rhinosinusitis and nasal polyps 2020. Rhinology. 2020;58(S29):1-464. doi:10.4193/Rhin 20.401

11. Lund VJ, Mackay IS. Staging in rhinosinusitis. Rhinology. 1993;31:183-184.

12. Heinzerling L, Mari A, Bergmann KC, et al. The skin prick test - European standards. Clin Transl Allergy. 2013;3(1):3. doi:10.1186/2045-7022-3-3

13. Lund VJ, Kennedy DW. Quantification for staging sinusitis. The staging and therapy group. Ann Otol Rhinol Laryngol. 1995;167:17-21. doi: $10.1177 / 000348949510410$ s02

14. Jang YJ, Koo TW, Chung SY, et al. Bone involvement in chronic rhinosinusitis assessed by $99 \mathrm{mTc}-\mathrm{MDP}$ bone SPECT. Clin Otolaryngol Allied Sci. 2002;27(3):156-161. doi:10.1046/j.1365-2273.2002.00553.x

15. Lee JT, Kennedy DW, Palmer JN, et al. The incidence of concurrent osteitis in patients with chronic rhinosinusitis: a clinicopathological study. Am J Rhinol. 2006;20(3):278-282. doi:10.2500/ajr.2006.20.2857

16. Hyo YK, Hun-Jong D, Hyun JL, et al. Hyperostosis may affect prognosis after primary endoscopic sinus surgery for chronic rhinosinusitis. Otolaryngol Head Neck Surg. 2006;135(1):94-99. doi:10.1016/j.otohns.2006.02.025

17. Ho J, Alvarado R, Rimmer J, et al. Atopy in chronic rhinosinusitis: impact on quality of life outcomes. Int Forum Allergy Rhinol. 2019;9(5):501507. doi:10.1002/alr.22272

18. Pearlman AN, Chandra RK, Chang D, et al. Relationships between severity of chronic rhinosinusitis and nasal polyposis, asthma, and atopy. Am $J$ Rhinol Allergy. 2009;23(2):145-148. doi:10.2500/ajra.2009.23.3284

19. Snidvongs K, McLachlan R, Chin D, et al. Osteitic bone: a surrogate marker of eosinophilia in chronic rhinosinusitis. Rhinology. 2012;50(3):299305. doi:10.4193/Rhino12.022

20. Zuo K, Guo J, Chen F, et al. Clinical characteristics and surrogate markers of eosinophilic chronic rhinosinusitis in Southern China. Eur Arch Otorhinolaryngol. 2014;271(9):2461-2468. doi:10.1007/s00405-014-2910-0

Journal of Inflammation Research

\section{Publish your work in this journal}

The Journal of Inflammation Research is an international, peer-reviewed open-access journal that welcomes laboratory and clinical findings on the molecular basis, cell biology and pharmacology of inflammation including original research, reviews, symposium reports, hypothesis formation and commentaries on: acute/chronic inflammation; mediators of inflammation; cellular processes; molecular mechanisms; pharmacology and novel anti-inflammatory drugs; clinical conditions involving inflammation. The manuscript management system is completely online and includes a very quick and fair peer-review system. Visit http://www.dovepress.com/testimonials.php to read real quotes from published authors.

Submit your manuscript here: https://www.dovepress.com/journal-of-inflammation-research-journal 